\title{
Associations between implementation of (1) crosmak Project Lazarus and opioid analgesic dispensing and buprenorphine utilization in North Carolina, 2009-2014
}

Apostolos A. Alexandridis ${ }^{1,2^{*}}$ (D), Nabarun Dasgupta ${ }^{1,2}$, Agnieszka D. McCort ${ }^{1}$, Christopher L. Ringwalt ${ }^{1}$, Wayne D. Rosamond ${ }^{2}$, Paul R. Chelminski ${ }^{3}$ and Stephen W. Marshall ${ }^{1,2}$

\begin{abstract}
Background: Project Lazarus (PL) is a seven-strategy, community-coalition-based intervention designed to reduce opioid overdose and dependence. The seven strategies include: community education, provider education, hospital emergency department policy change, diversion control, support programs for patients with pain, naloxone policies, and addiction treatment expansion. PL was originally developed in Wilkes County, NC. It was made available to all counties in North Carolina starting in March 2013 with funding of up to $\$ 34,400$ per county per year. We examined the association between PL implementation and 1) overall dispensing rate of opioid analgesics, and 2) utilization of buprenorphine. Buprenorphine is often used in connection with medication assisted treatment (MAT) for opioid dependence.
\end{abstract}

Methods: Observational interrupted time series analysis of 100 counties over 2009-2014 ( $n=7200$ county-months) in North Carolina. The intervention period was March 2013-December 2014. 74 of 100 counties implemented the intervention. Exposure data sources comprised process surveys, training records, Prescription Drug Monitoring Program (PDMP) data, and methadone treatment program quality data. Outcomes were PDMP-derived counts of opioid prescriptions and buprenorphine patients. Incidence Rate Ratios were estimated with adjusted GEE Poisson regression models of all seven PL strategies.

Results: In adjusted models, diversion control efforts were positively associated with increased dispensing of opioid analgesics (IRR: 1.06; 95\% Cl: 1.03, 1.09). None of the other PL strategies were associated with reduced prescribing of opioid analgesics. Support programs for patients with pain were associated with a non-significant decrease in buprenorphine utilization (IRR: 0.93; 95\% Cl: 0.85, 1.02), but addiction treatment expansion efforts were associated with no change in buprenorphine utilization (IRR: 0.98; $95 \%$ Cl: 0.91, 1.06).

Conclusions: Implementation of PL strategies did not appreciably reduce opioid dispensing and did not increase buprenorphine utilization. These results are consistent with previous findings of limited impact of PL strategies on overdose morbidity and mortality. Future studies should analyze the uptake of MAT using a more expansive view of institutional barriers, treating community coalition activity around MAT as an effect modifier.

Keywords: Opioids, Overdose, Buprenorphine, MAT, Community coalitions, Prevention, PDMP, Interrupted time series, Evaluation

\footnotetext{
* Correspondence: aaa@unc.edu

${ }^{1}$ Injury Prevention Research Center, University of North Carolina at Chapel

Hill, Chapel Hill, North Carolina, USA

${ }^{2}$ Department of Epidemiology, Gillings School of Global Public Health,

University of North Carolina at Chapel Hill, Chapel Hill, North Carolina, USA

Full list of author information is available at the end of the article
}

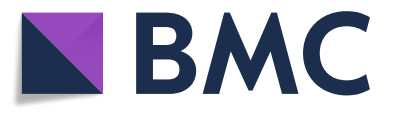

(c) The Author(s). 2019 Open Access This article is distributed under the terms of the Creative Commons Attribution 4.0 International License (http://creativecommons.org/licenses/by/4.0/), which permits unrestricted use, distribution, and reproduction in any medium, provided you give appropriate credit to the original author(s) and the source, provide a link to the Creative Commons license, and indicate if changes were made. 


\section{Background}

Deaths from opioid overdose began increasing in North Carolina (NC) in the late 1990s (Web-based Injury Statistics Query and Reporting System (WISQARS), 2005). Between 1999 and 2015, opioid mortality increased 486\% to over 11 per 100,000 (Injury and Epidemiology Surveillance Unit, Injury and Violence Prevention Branch, Division of Public Health, North Carolina Department of Health and Human Services, 2015). Opioid overdose has become the leading cause of unintentional injury death in the state, and involves prescription opioid analgesics (OA) as well as illicitly manufactured heroin and fentanyl (State Center for Health Statistics, 2015). Addressing this epidemic has become a leading priority for the NC Department of Health and Human Services (NC DHHS), which has promoted supply, demand, and harm reduction strategies (North Carolina Department of Health and Human Services, 2017).

Among demand reduction strategies, medication assisted treatment (MAT), particularly with the partial opioid agonist buprenorphine, has been widely embraced. MAT is supported by substantial evidence-based modalities of substance abuse treatment (Mattick et al., 2014; Thomas et al., 2014). Buprenorphine is the only form of agonist MAT that can be dispensed by traditional retail pharmacies, and can be prescribed by primary care providers who complete an 8-h training through the Substance Abuse and Mental Health Services Administration (SAMHSA) (Fiellin et al., 2004). Buprenorphine also has advantages for patients seeking agonist-based MAT in rural areas (Kraus et al., 2011). Formulations of buprenorphine indicated for MAT are also often used to reduce the risk of opioid abuse in patients receiving high doses of full-agonist opioids.

Project Lazarus (PL) is a comprehensive, communitybased series of seven interventions designed to reduce demand, supply and harms related to prescription OA; improve treatment of chronic pain; and promote and improve access to MAT. PL was first piloted in one NC county between 2007 and 2010, and was implemented statewide in early 2013 (Albert et al., 2011). Subsequently, the seven distinct PL strategies were promoted nationally by the White House Office of National Drug Control Policy (ONDCP) 2015 opioid strategy (United States, 2015). Funding for coalitions was made available to all $100 \mathrm{NC}$ counties through a non-competitive application process organized by the state Medicaid implementation authority, Community Care of North Carolina (CCNC), and the Mountain Area Health Education Center (MAHEC). Coalitions were invited to select among the seven PL strategies they felt best represented their community's needs, with a minimum of three.

PL's seven distinct strategies are designed to be implemented together by a community-based coalition. This paper examines the association between the seven PL strategies and (1) overall prescribing rate of opioid analgesics and (2) utilization of buprenorphine. The $7 \mathrm{PL}$ strategies are as follows. (1) Community education promoted public awareness of prescription opioid overdose. (2) Diversion control was designed to remove unused medications and train law enforcement on OA diversion. (3) Support programs for patients with pain provided support groups, case management and pain clinic vetting and referrals. (4) Provider education focused on educating medical professionals in chronic pain treatment, including group trainings and in-office 'academic detailing, or tailored instruction. The North Carolina Medical Board's published guidelines for pain management were referenced in trainings (Trado, 2004). (5) Hospital emergency department (ED) policies revised hospital practices to limit ED OA prescribing and require checking the state's Prescription Drug Monitoring Program (PDMP) before prescribing. (6) Addiction treatment expansions increased the number of providers in a community able to prescribe buprenorphine-based MAT for opioid dependence, and the number of beds available in inpatient detoxification and treatment facilities. (7) Naloxone policies promoted liberal distribution of the opioid antagonist naloxone to opioid users and their close contacts, first responders including EMS and police, and caregivers. Strategies 1-3 were focused on community entities external to the health care system, whereas strategies 4-7 were focused on health care providers (Table 1).

The statewide implementation of PL in NC has significance as one of the earliest and largest coordinated efforts to address the overdose epidemic using community-based approaches. We hypothesized that the seven PL strategies would have varying effects on opioid overdose morbidity and mortality, opioid prescribing, and utilization of buprenorphine (Table 1). This paper focuses on PL's hypothesized effects on opioid prescribing and buprenorphine utilization. An evaluation of the association between PL and opioid overdose morbidity and mortality has appeared elsewhere (Alexandridis et al., 2018).

\section{Methods}

$\mathrm{NC}$ is a large state in the southeastern US (population 9.9 million in 2014) that had overdose rates comparable to the US average during the 2009-2014 study period. We used an interrupted time series design to examine the relationship between strategies implemented as a part of PL and both prescription OA dispensing and buprenorphine utilization rates.

The general analytic approach has been described previously (Alexandridis et al., 2018). Primary and administrative secondary data sources were aggregated at the level of the county for every month over the time period 2009- 
Table 1 Project Lazarus strategies and hypothesized effects

\begin{tabular}{|c|c|c|c|c|}
\hline & Strategy & Examples of Key Activities & $\begin{array}{l}\text { Expected effect on } \\
\text { opioid analgesic } \\
\text { prescribing }\end{array}$ & $\begin{array}{l}\text { Expected effect } \\
\text { on buprenorphine } \\
\text { utilization }\end{array}$ \\
\hline \multirow[t]{2}{*}{1} & \multirow[t]{2}{*}{ Community Education } & - Community- and school-based prevention education & \multirow[t]{2}{*}{ Decrease } & \multirow[t]{2}{*}{ Increase } \\
\hline & & - Multi-media advertising campaigns & & \\
\hline \multirow[t]{3}{*}{2} & \multirow[t]{3}{*}{ Diversion Control } & - Education on proper storage of medications & \multirow[t]{3}{*}{ No change } & \multirow[t]{3}{*}{ No change } \\
\hline & & - Pill take-backs and fixed disposal sites & & \\
\hline & & $\begin{array}{l}\text { - Training of law enforcement in the prevention of } \\
\text { medication diversion and the arrest of diverters }\end{array}$ & & \\
\hline \multirow[t]{3}{*}{3} & \multirow[t]{3}{*}{$\begin{array}{l}\text { Support for Patients with } \\
\text { Pain }\end{array}$} & $\begin{array}{l}\text { - Development of support groups and other extra-clinical } \\
\text { services for patients }\end{array}$ & \multirow[t]{3}{*}{ Decrease } & \multirow[t]{3}{*}{ Increase } \\
\hline & & - Referrals of patients to clinics & & \\
\hline & & - Pain patient education on reducing risks of overdose & & \\
\hline \multirow[t]{2}{*}{4} & \multirow[t]{2}{*}{ Provider Education } & $\begin{array}{l}\text { - Continuing education on the effective management of } \\
\text { chronic pain and appropriate opioid prescribing }\end{array}$ & \multirow[t]{2}{*}{ Greatest decrease } & \multirow[t]{2}{*}{ Increase } \\
\hline & & - Effective management of patients with chronic pain & & \\
\hline \multirow[t]{2}{*}{5} & \multirow{2}{*}{$\begin{array}{l}\text { Hospital Emergency } \\
\text { Department (ED) Policy }\end{array}$} & - Policies to limit controlled substance dispensing in EDs & \multirow[t]{2}{*}{ Decrease } & \multirow[t]{2}{*}{ No change } \\
\hline & & - Policies to require use of the PDMP in EDs & & \\
\hline \multirow[t]{2}{*}{6} & \multirow[t]{2}{*}{ Addiction Treatment } & $\begin{array}{l}\text { - Increases in availability/access to drug detoxification programs } \\
\text { and treatment clinics }\end{array}$ & \multirow[t]{2}{*}{ No change } & \multirow[t]{2}{*}{ Greatest increase } \\
\hline & & $\begin{array}{l}\text { - Increases in the number of providers authorized to prescribe buprenorphine } \\
\text { for addiction }\end{array}$ & & \\
\hline \multirow[t]{2}{*}{7} & \multirow[t]{2}{*}{ Naloxone Policies } & $\begin{array}{l}\text { - Provision of naloxone to patients and nonmedical opioid } \\
\text { users with high overdose risk and their family members }\end{array}$ & \multirow[t]{2}{*}{ No change } & \multirow[t]{2}{*}{ No change } \\
\hline & & - Education on reversing overdose before EMS arrival & & \\
\hline
\end{tabular}

2014. These secondary data sources included the state PDMP and drug treatment intake interviews. The resulting time series captured relevant activities of PL coalition activities and opioid-related outcomes across a total of 7200 county-months.

\section{Implementation of PL strategies}

PL strategies were implemented by a series of county-based community coalitions. Funding for the intervention was made available to all $100 \mathrm{NC}$ counties via an application process for county-based coalitions beginning in 2011. Funding was distributed through CCNC (the designated state Medicaid implementation authority) and MAHEC, with technical support from the community-based organization Project Lazarus. Coalitions that applied received annual grants of between $\$ 6500$ and $\$ 34,400$, from a network of funding sources. Thus, a coalition that received the maximal funding $(\$ 34,440)$ may have been able to provide a full-time salary for a community health worker paid at the average weekly wage in North Carolina. Given that additional coordinators and non-personnel costs would be needed to successfully implement the seven strategies, it is reasonable to assume that no county received funding sufficient to fully implement PL without the need for additional investment by the county or community.
Our evaluation included a pre-intervention period (January 2009-February 2013) and an intervention period (March 2013-December 2014). CCNC also funded Medicaid regional coordinators who provided technical assistance to community coalitions, directed provider education, and advocated for changes in hospital policies related to opioid prescribing.

We used measures of coalition activities and ongoing surveys of key community coalition leaders to capture the implementation of the $7 \mathrm{PL}$ strategies in each county in each month. We coded implementation of PL strategies using dichotomous variables that captured the implementation of each strategy, with ' 0 ' representing no implementation of a strategy in a county to-date, and ' 1 ' representing any ongoing or prior implementation or policy change specific to each strategy.

Community-based coalitions were identified the time they were funded by CCNC. Coalition activities were captured through structured surveys that three of the authors (ADM, ND, CLR) administered via web survey every 6 months to coalition leaders and the CCNC regional coordinators. Surveys included details on naloxone policy adoption, ED policy changes, creation of support programs for patients with pain, and the location and date of provider and community education events. 
For the diversion control strategy, details of the time and location of local law enforcement trainings on diversion control were obtained from the NC State Bureau of Investigation (SBI).

For the addiction treatment strategy and the evaluation of PL association with opioid dispensing, we combined survey data on MAT expansions with measures of incident buprenorphine and methadone utilization. This measure was constructed with data from the NC Controlled Substance Reporting System (CSRS), the state PDMP, and the NC Treatment Outcomes and Program Performance System (NC-TOPPS), a quality monitoring system sponsored by the Substance Abuse and Mental Health Services Administration (SAMHSA). Overall counts of new methadone treatment program patients were abstracted from intake interviews, and added to measures of incident MAT. Buprenorphine treatment episodes were considered incident after a 90-day washout period since the last buprenorphine script dispensed. The evaluation of PL's association with buprenorphine utilization only used the former survey data on MAT expansions and policy change.

\section{Opioid prescribing and buprenorphine utilization}

Data from the CSRS were used to construct countymonth counts of patients and prescriptions for opioid analgesics. PDMPs such as the CSRS are state government-run electronic databases that can be queried at the point of care by clinicians to review a patient's history of receiving controlled substances. Selected law enforcement officers and medical examiners are allowed access to the database when they are investigating specific cases. The CSRS began collecting data in January 2009, and data were provided by the NC Division of Mental Health, Developmental Disabilities, and Substance Abuse Services (DMHDDSAS). The data are generated when prescriptions for controlled substances are dispensed at regulated pharmacies in North Carolina. The data captured comprise each field of information legally required to be included in a North Carolina prescription for a controlled substance. The data are stored locally at the pharmacy and transmitted periodically to a central database. Data elements include unique identifiers for prescribers, dispensers, and patients and their locations; quantity, dose, days supply, and National Drug Code of the prescription; and age and sex of the patient.

The raw data were tabulated by active pharmaceutical ingredient (API) and dosage form (e.g., solid oral, patch) for opioid analgesics. Opioid analgesics were defined as solid oral, transbuccal, or transdermal formulations containing codeine, fentanyl, hydrocodone, hydromorphone, methadone, morphine, oxycodone and oxymorphone. Prescriptions with APIs comprising the top 99.9\% of all prescription records were retained; data cleaning removed non-controlled substances and appended metadata on drug class. Patients were assigned a unique identification number provided by the database vendor (Health Information Designs, Auburn, Alabama, USA), which takes name, date of birth, and residential ZIP code into account, and was provided as a one-way hash algorithm and was continuous over data-years.

Data from the CSRS were used to create countymonth counts for the two outcome measures of interest, opioid prescribing and buprenorphine utilization. For opioid prescribing, counts of dispensed full muopioid receptor (MOR) agonist analgesics and their prodrugs, including solid oral, transdermal, nasal spray, and transbuccal formulations, were extracted by county and month from the CSRS. For buprenorphine utilization, counts of unique monthly buprenorphine patients, created using prescription data from the CSRS, were used to identify all patients receiving pharmacy-dispensed formulations of buprenorphine with indications for addiction treatment (e.g. Subutex, Suboxone, but not Butrans).

\section{Covariate measures}

In order to control for fundamental differences in health status between counties (e.g., "healthy county effect") and over time, a construct from the Robert Wood Johnson Foundation (RWJF) County Health Rankings was used (Remington et al., 2015). This "county health factors" variable is a composite Z-score-based ranking which comprises health behaviors, including tobacco use, diet and exercise, alcohol and drug use, and sexual activity; clinical care, including access to and quality of care; social and economic factors, including education, employment, income, family and social support, and community safety; and physical environment, including air and water quality, housing and transit. This was available for 2010 onwards; for 2009 the data from 2010 to 2016 were used to linearly extrapolate county months. Annual data were linearly interpolated to generate county-month scores. This score was used in fully-adjusted, immediate-effect multivariable models of all seven strategies.

\section{Statistical methods}

We used incidence rate ratios (IRRs) to quantify the association between the each of the seven PL strategies and our two outcomes, and used Poisson regression to model the IRRs. Models were fit using generalized estimating equations (GEE) to account for clustering at the county level, with a population offset for each county-month (Alexandridis et al., 2018). These models were assessed for indicators of overdispersion, and Negative Binomial (NB2) models were also assessed. 
For opioid dispensing and buprenorphine utilization separately, our models were defined as:

$$
\begin{aligned}
\lambda=\ln (\mathrm{a} / \mathrm{n})=\ln (\mathrm{a})-\ln (\mathrm{n})= & \beta_{0}+\left[\beta_{1} \mathrm{X}_{1}+\cdots\right. \\
& \left.+\beta_{7} \mathrm{X}_{7}\right]+\left[\beta_{8} \mathrm{X}_{8}\right] \\
& +\left[\beta_{9} \mathrm{X}_{9}\right]+\left[\beta_{10} \mathrm{X}_{10}\right] \\
& +\left[\beta_{11} \mathrm{X}_{11} \ldots \beta_{13} \mathrm{X}_{13}\right] \\
& +[-\ln (\mathrm{n})]
\end{aligned}
$$

where $\lambda$ was a rate of opioid dispensing or buprenorphine utilization per county-month residential population, $a$ was a count of opioid dispensing or buprenorphine utilization, and $\beta_{0}$ is the intercept.

$\mathrm{X}_{1}, \ldots, \mathrm{X}_{7}$ were the independent (exposure) variables that designate the presence or absence of the seven intervention strategies for any given month and county. Each strategy was represented as dichotomous variable with no implementation as the referent (coded 0), and implementation (coded 1).

$\mathrm{X}_{8}$ was the county-month rate of outpatient prescriptions dispensed for opioid analgesics in units of 1000 , with total resident population as the denominator. This measure was only used as a covariate in models of buprenorphine utilization.

$\mathrm{X}_{9}$ is the county health status variable of linearly interpolated annual z-scores of Health Factors from RWJF County Health Rankings. This variable was used as a marker for general community health status and was included to control for potential confounding by changes to general community health status over time and between counties.

$\mathrm{X}_{10}$ was a variable for calendar year included to remove linear trends over time ("secular trend").

$\mathrm{X}_{11}, \mathrm{X}_{12}$, and $\mathrm{X}_{13}$ were indicator variables for seasonality, implemented with indicator coding for spring, summer and fall, with winter as the referent. These variables are included to de-trend for seasonal effects on overdose and related outcomes, which were observed in preliminary data analysis. For opioid overdoses in 2010, a Walter and Elwood analysis of seasonality using the exact method test suggested the presence of seasonality (chi-square 5.8, $p=$ $0.05,2 \mathrm{df}$ ) with a peak in March.

The offset term $\ln (n)$ was the $\log _{e}$ denominator of the rates, defined as the resident population of the county. Annual population was obtained from the National Center for Health Statistics and linearly interpolated by month.

Goodness-of-fit was assessed using Akaike and Bayesian Information Criterion (AIC and BIC), with smaller values indicating better fit relative to an intercept-only model. We assessed over-dispersion using Deviance and Pearson's chi-square divided by its degrees of freedom. Initially, univariate models were used to examine each of the individual strategies, without any adjustment for trends by year and season, county health status, or other strategies. Multivariable adjusted models examined the associations for each of the seven strategies while controlling for the other six strategies, with additional adjustment for year and season, and county health factors. Adjusted models of buprenorphine utilization also included adjustment for each county's population-based rate of OA prescribing.

\section{Results}

A total of 74 out of $100 \mathrm{NC}$ counties implemented any strategy of PL by the end of the intervention period, covering $70 \%$ of the state population. Non-implementing counties were either ineligible due to a lack of resources or did not submit a funding application.

Over the 2009-2014 study period, unique annual OA patients decreased by $6.9 \%$, from $23.0 \%$ of all state residents in 2009 to $21.4 \%$ in 2014 (Alexandridis et al., 2018). Annual prescriptions dispensed for OA increased by $17.3 \%$, from 6.22 million to 7.30 million, an $11.4 \%$ increase (0.66 to 0.73 per person-year). The most commonly dispensed OAs were hydrocodone, oxycodone, codeine and morphine.

\section{Opioid analgesic dispensing}

In univariate models (no adjustment), we found weak associations between the adoption of PL strategies and the rate of OA prescription dispensing (Table 2). MAT expansion was associated with a $16 \%$ increase in OA dispensing (IRR: 1.16; 95\% CI: 1.11, 1.20), and Diversion Control efforts were associated with a $15 \%$ increase (IRR: 1.15; 95\% CI: 1.12, 1.17) in OA dispensing.

In fully-adjusted multivariable models accounting for implementation of all seven strategies, year and season, and county health status, these associations were attenuated. A statistically significant association between $\mathrm{Di}$ version Control strategies and increased prescribing persisted (IRR: 1.06; 95\% CI: 1.03, 1.09). No other strategy was associated with a $5 \%$ or higher increase or decrease in opioid prescribing. Notably, the strategy of Provider Education was not associated with any change in OA dispensing (IRR: 1.00; 95\% CI: 0.97, 1.03).

\section{Buprenorphine utilization}

In univariate models (no adjustment), each PL strategy was associated with a $54-82 \%$ increase in the rate of buprenorphine utilization (Table 3). After adjustment for time and season, these associations were greatly attenuated in single-strategy models; in fact, support programs for patients with pain were associated with a $15 \%$ decrease in buprenorphine (IRR: 0.85; 95\% CI: 0.78, 0.93).

In fully-adjusted multivariable models including all seven PL strategies, only support programs for patients with pain were associated with a change of $5 \%$ or greater 
Table 2 Associations between Project Lazarus implementation and opioid analgesic prescribing, by strategy, North Carolina, 20092014

\begin{tabular}{|c|c|c|c|c|c|c|c|c|c|}
\hline \multirow[b]{2}{*}{ Strategy } & \multirow[b]{2}{*}{ Implementation Level } & \multirow[b]{2}{*}{ County-months } & \multirow[b]{2}{*}{ Rx OAs dispensed } & \multicolumn{3}{|c|}{ Univariate Models $^{\mathrm{a}}$} & \multicolumn{3}{|c|}{ Multivariable Adjusted Model } \\
\hline & & & & $\mathrm{IRR}$ & $95 \% \mathrm{Cl}$ & CLR & $\mathrm{IRR}$ & $95 \% \mathrm{Cl}$ & CLR \\
\hline \multirow[t]{2}{*}{ Diversion Control } & None & 4971 & $23,512,894$ & 1 (ref.) & & & 1 (ref.) & & \\
\hline & Any & 2229 & $17,848,868$ & 1.15 & $1.12,1.17$ & 1.044 & 1.06 & $1.03,1.09$ & 1.056 \\
\hline \multirow[t]{2}{*}{ Naloxone Policies } & None & 6216 & $34,103,605$ & 1 (ref.) & & & 1 (ref.) & & \\
\hline & Any & 984 & $7,258,157$ & 1.08 & $1.06,1.11$ & 1.038 & 0.97 & $0.95,0.99$ & 1.048 \\
\hline \multirow[t]{2}{*}{ Community Education } & None & 5969 & $30,353,079$ & 1 (ref.) & & & 1 (ref.) & & \\
\hline & Any & 1231 & $11,008,683$ & 1.11 & $1.09,1.13$ & 1.042 & 1.00 & $0.97,1.03$ & 1.058 \\
\hline \multirow[t]{2}{*}{ Provider Education } & None & 4962 & $25,608,213$ & 1 (ref.) & & & 1 (ref.) & & \\
\hline & Any & 2238 & $15,753,549$ & 1.13 & $1.10,1.15$ & 1.042 & 1.00 & $0.97,1.03$ & 1.060 \\
\hline \multirow[t]{2}{*}{ Support for Patients with Pain } & None & 6684 & $34,023,002$ & 1 (ref.) & & & 1 (ref.) & & \\
\hline & Any & 516 & $7,338,760$ & 1.08 & $1.06,1.10$ & 1.029 & 0.96 & $0.93,1.00$ & 1.069 \\
\hline \multirow[t]{2}{*}{ Hospital ED Policy } & None & 5485 & $29,009,970$ & 1 (ref.) & & & 1 (ref.) & & \\
\hline & Any & 1715 & $12,351,792$ & 1.11 & $1.08,1.13$ & 1.047 & 0.99 & $0.96,1.01$ & 1.055 \\
\hline \multirow[t]{2}{*}{ Addiction Treatment } & None & 1559 & $13,442,436$ & 1 (ref.) & & & 1 (ref.) & & \\
\hline & Any & 5641 & $27,919,326$ & 1.16 & $1.11,1.20$ & 1.085 & 1.04 & $0.99,1.08$ & 1.090 \\
\hline
\end{tabular}

IRR incidence rate ratio, $C I$ confidence interval, $C L R$ confidence limit ratio, ED Emergency Department

Results from Poisson GEE with county-month population offset

annivariate unadjusted models for each strategy

${ }^{\mathrm{b}}$ Adjusted multivariable model adjusts for the other six strategies, county health status, year and season

in buprenorphine use (IRR: 0.93; 95\% CI: 0.85, 1.02), and no strategy was associated with a statistically significant change. The addiction treatment strategy hypothesized to have a direct impact on this outcome was associated with a $2 \%$ reduction in buprenorphine use (IRR: 0.98; 95\% CI: 0.91, 1.06). An additional model of only the addiction treatment strategy (including adjustment for county health status in addition to year and season, but without the other six strategies) found no association (IRR: 1.00; 95\% CI: 0.92, 1.09).

\section{Discussion}

Project Lazarus was implemented statewide in NC as a community-based program with multi-agency support. Its goals were to address opioid supply, demand, and harm reduction. PL sought to improve access to MAT and reduce opioid prescribing, while maintaining legitimate access to opioids for patients with chronic pain. The results of this analysis, together with our previous analysis of the association between PL and overdose morbidity and mortality, indicate that implementation of the PL strategies neither appreciably reduced opioid dispensing nor increased buprenorphine utilization (Alexandridis et al., 2018).

For a community-coalition-based program such as PL to be successful, as was observed in the pilot implementation in Wilkes County, NC, a strong community-public health partnership needs to be established (Albert et al., 2011). Indicators of a strong partnership include sustained and focused engagement by a local health department or similar public health agency with health care provider networks and/or key enforcement agencies, such as local law enforcement (Alexandridis et al., 2017). Such partnerships are relatively uncommon in communities, particularly around the issue of substance use, pain, opioids, or overdose. Local Health Departments offer a potential starting point for a coalition to crystalize around, but deep engagement with stakeholders outside of the local public health infrastructure is also critical (Alexandridis et al., 2017). The maximal annual funding, less than $\$ 35,000$, provided to the PL coalitions was insufficient for the hiring of full-time community health worker organizers with sufficient budgets for implementation activity. Even if funding were sufficient to hire full-time employees, the motivation for various activities must also be internal to the community to achieve the greatest sustained effect. It is possible that we may have seen a greater effect from the statewide PL program if both funding levels and community readiness to implement actions based on the PL model had been at higher levels.

\section{Opioid prescribing}

Diversion control efforts were the lone PL strategy associated with a statistically significant, $6 \%$ increase in opioid dispensing. Though unanticipated in its direction, this association was not clinically significant in its magnitude. Given the consistently high reported levels of unused controlled substances (CS) sharing between friends and family 
Table 3 Associations between Project Lazarus implementation and buprenorphine utilization, by strategy, North Carolina, 2009-2014

\begin{tabular}{|c|c|c|c|c|c|c|c|c|c|}
\hline \multirow[b]{2}{*}{ Strategy } & \multirow[b]{2}{*}{ Implementation Level } & \multirow[b]{2}{*}{ County-months } & \multirow[b]{2}{*}{ Monthly Bup. Patients } & \multicolumn{3}{|c|}{ Univariate Models $s^{a}$} & \multicolumn{3}{|c|}{$\begin{array}{l}\text { Multivariable Adjusted } \\
\text { Model }^{b}\end{array}$} \\
\hline & & & & IRR & $95 \% \mathrm{Cl}$ & $\mathrm{CLR}$ & $\mathrm{IRR}$ & $95 \% \mathrm{Cl}$ & $\mathrm{CLR}$ \\
\hline \multirow[t]{2}{*}{ Diversion Control } & None & 4971 & 266,389 & 1 (ref.) & & & 1 (ref.) & & \\
\hline & Any & 2229 & 323,576 & 1.82 & $1.71,1.93$ & 1.128 & 1.00 & $0.93,1.08$ & 1.165 \\
\hline \multirow[t]{2}{*}{ Naloxone Policies } & None & 6216 & 443,181 & 1 (ref.) & & & 1 (ref.) & & \\
\hline & Any & 984 & 146,784 & 1.67 & $1.57,1.78$ & 1.133 & 1.02 & $0.96,1.08$ & 1.122 \\
\hline \multirow[t]{2}{*}{ Community Education } & None & 5969 & 379,482 & 1 (ref.) & & & 1 (ref.) & & \\
\hline & Any & 1231 & 210,483 & 1.68 & $1.59,1.78$ & 1.117 & 0.98 & $0.91,1.05$ & 1.164 \\
\hline \multirow[t]{2}{*}{ Provider Education } & None & 4962 & 303,822 & 1 (ref.) & & & 1 (ref.) & & \\
\hline & Any & 2238 & 286,143 & 1.77 & $1.68,1.87$ & 1.117 & 1.00 & $0.93,1.07$ & 1.148 \\
\hline \multirow[t]{2}{*}{ Support for Patients with Pain } & None & 6684 & 454,973 & 1 (ref.) & & & 1 (ref.) & & \\
\hline & Any & 516 & 134,992 & 1.54 & $1.45,1.64$ & 1.131 & 0.93 & $0.85,1.02$ & 1.200 \\
\hline \multirow[t]{2}{*}{ Hospital ED Policy } & None & 5485 & 360,241 & 1 (ref.) & & & 1 (ref.) & & \\
\hline & Any & 1715 & 229,724 & 1.68 & $1.57,1.80$ & 1.146 & 0.98 & $0.92,1.04$ & 1.134 \\
\hline \multirow{2}{*}{$\begin{array}{l}\text { Addiction Treatment } \\
\text { (Policy Only) }\end{array}$} & None & 6549 & 512,646 & 1 (ref.) & & & 1 (ref.) & & \\
\hline & Any & 651 & 77,319 & 1.62 & $1.51,1.74$ & 1.148 & 0.98 & $0.91,1.06$ & 1.161 \\
\hline
\end{tabular}

IRR incidence rate ratio, $C l$ confidence interval, $C L R$ confidence limit ratio, ED Emergency Department

Results from Poisson GEE with county-month population offset

anivariate unadjusted models for each strategy

${ }^{\mathrm{b}}$ Adjusted multivariable model adjusts for the other six strategies, county opioid analgesic prescribing rate, county health status, year and season

as reported by national data (Lipari \& Hughes, 2017), one possible explanation is that aggressive take-back and drop-box efforts have led to modest increases in people seeking opioid prescriptions (Lewis et al., 2014; Wakeland et al., 2015). Likewise, other forms of anti-diversion law enforcement activity may have led to increases in the seeking of legitimate opioid prescriptions. It is also possible that this result is due to bias resulting from a misclassification of exposure in law enforcement trainings, which were a component of the diversion control strategy. The SBI targeted known areas of high opioid diversion activity for their trainings, which were in turn attended by law enforcement officers from multiple counties. As we were only able to capture the counties where trainings occurred, it is possible that counties were uncredited for the implementation of this strategy.

A previous study in Massachusetts demonstrated a significant decline in opioid prescribing and unique opioid patients after a comprehensive opioid and pain policy was adopted by a large statewide private insurer (Garcia et al., 2016). Our null result highlights potential limitations of diffuse community coalitions to create significant changes in prescriber practice as compared to a centralized, insurance-directed approach. The lack of impact of PL statewide implementation on prescribing may potentially reflect insufficient investment in local coalition activities. Additionally, it is important to note that PL, as implemented statewide in $\mathrm{NC}$, was not designed with an explicit focus on reducing opioid dispensing volumes, but rather promoting appropriate pain management. The community-facing supply reduction efforts of PL focused on the prevention of prescription opioid sharing through unused drug disposal and education, whereas the healthcare-facing efforts addressed acute opioid prescribing in EDs and chronic pain treatment among community-based physicians. Only these latter physician education strategies would be expected to have a direct effect on opioid prescribing; however, a reduction was not observed in this study.

It is also likely that the effectiveness of PL activities to limit prescribing were affected by the changing pace and form of the overdose epidemic in the US during the implementation period. When PL was initially piloted in Wilkes County, NC, through the planning of the statewide implementation, it was not anticipated that nested epidemics of heroin and fentanyl overdose would occur at the scale since documented (Ciccarone, 2017; Unick et al., 2013; Cicero et al., 2015). At the time of the implementation, there was evidence that an inflection point in the epidemic had been reached (Dart et al., 2015a; Dart et al., 2015b). Future community-based efforts to reduce overdose must have the capacity to respond rapidly to evolving patterns of substance use and develop a priori contingency plans. One potential tool that state or federal agencies could use to identify motivated communities is the Community Readiness scale developed by the Tri-Ethnic Center for Prevention Research (Ringwalt et al., 2018). A multi-stage process could first identify communities with high motivation and infrastructure to deploy a community-based program, and target them to implement 
a PL-like program, while simultaneously developing motivation and infrastructure using other approaches elsewhere.

\section{Buprenorphine utilization}

We found no strong association between any component of PL and buprenorphine utilization in adjusted models. Unadjusted univariate models indicated consistent increases in the utilization rate, even for strategies not expected to have a direct impact on buprenorphine, which were hypothesized to be the result of secular trends in MAT over the study period.

In our previous study, we found the PL addiction treatment strategy was associated with increased overdose mortality (Alexandridis et al., 2018). Together with the findings presented here, this suggests that areas with high MAT utilization were not necessarily influenced by PL, as PL-related MAT policy changes were not associated with a change in the rate of buprenorphine utilization. We focused on buprenorphine specifically because of its advantages in the management of opioid use disorder in rural areas (Kraus et al., 2011), and because buprenorphine's non-MAT use remains closely linked to the clinical management of patients with high risks of opioid dependence or use disorder (Fiellin et al., 2014; Blondell et al., 2010).

It is important to note that even buprenorphine MAT requires a substantial investment to reduce fatal overdose. National surveillance of buprenorphine and heroin overdose in France, where buprenorphine accounts for well over $80 \%$ of all MAT, found an $82 \%$ reduction in heroin overdose deaths between 1995 and 2003 after the introduction of community-based MAT through primary care providers and community pharmacies in 1996 (Emmanuelli \& Desenclos, 2005; Carrieri et al., 2006). However, total MAT utilization increased 100-fold nationally in that time period, and each prevented death was associated with upwards of 200 MAT patients. Within the US, the training requirement and patient limits imposed by the Drug Addiction Treatment Act of 2000 provide additional challenges to the effective implementation of buprenorphine-based MAT, due to incomplete coverage of MAT costs among Medicare/ Medicaid patients (Knudsen et al., 2011). People in the custody of the criminal justice system also face considerable restrictions on access to MAT, particularly effective agonist-based MAT, as its use is mediated by drug court staff, judges, correctional facilities, and local and state politics (Friedmann et al., 2012; Brinkley-Rubinstein et al., 2017). These challenges and barriers to treatment underscore the difficulties faced in moving beyond simple supply reduction approaches to community-based addiction treatment (Dasgupta et al., 2018).
Recent strategy recommendations, such as the President's Commission Report, have heavily stressed substance abuse treatment expansion, particularly maintaining access to MAT (Christie et al., 2017). Highly motivated and effective community coalitions are only able to expand or maintain such MAT programs when they are supported or endorsed by diverse federal and state entities, such as Medicaid/Medicare; justice departments, drug courts, and correctional systems; and SAMHSA and its state-level counterparts. Stigma and resistance to agonist MAT affects all levels of this structure, and coalition activity may have a limited impact on local attitudes and views (Ringwalt et al., 2018). Future studies should analyze the uptake of MAT using a more expansive view of these institutional barriers, treating coalition and community activity with regards to MAT as an effect modifier of state and federal policies.

\section{Limitations}

Our evaluation of PL was limited by funder priorities that all North Carolina counties should implement PL, necessitating an observational interrupted time series study design. We were therefore unable to randomize communities to receive PL funding and supports, and residual or uncontrolled confounding may be present. The associations between PL strategies and our outcomes cannot be interpreted as causal. In particular, we were unable to quantify any of the selection factors associated with higher intensity of PL implementation in a given community. Multiple factors influencing coalition activity and substance use could not be obtained with the appropriate spatial and temporal resolution, including: previous collaborations among stakeholders (Kegler et al., 2010), external measures of coalition leadership (Kegler et al., 1998), private insurance policy changes (Garcia et al., 2016), prescriber utilization of the PDMP (Delcher et al., 2015), and the implementation of the Risk Evaluation and Mitigation Strategies (REMS) for transmucosal immediate-release fentanyl and extended-release/long-acting opioid analgesics (Food and Drug Administration, 2012; Cepeda et al., 2017). All were therefore assumed to have nondifferential effects. Because the REMS were not fully implemented by the end of the intervention period their likely effect was minimal. Our model of the intervention also assumes that implementation of PL strategies occurs with high fidelity and that all the PL strategies have a sustained ongoing effect, or are continuously implemented. These are strong assumptions to make in the context of community coalition-based programs funded at relatively modest levels. Finally, although we did not detect changes in overall volume of prescribing, it is possible that the nature of prescribing was altered and inappropriate prescribing was reduced. 
Outcomes for both analyses in this study were derived from PDMP data, which have the typical caveats of administrative, secondary data. Our ability to identify unique buprenorphine patients is limited by the proprietary entity resolution algorithms used to link prescriptions based on name, address, and date of birth, potentially more challenging in vulnerable populations that may be more geographically mobile (Galea \& Vlahov, 2002). Unlinked records would result in overestimations of unique patients; we addressed this possible source of bias by using a prevalent rather than incident patient outcome.

Finally, our post-intervention period was limited to 22 months. The original pilot of PL in Wilkes County saw its greatest effect after three years of implementation (Albert et al., 2011). Future evaluations of communitybased approaches to overdose should consider the length of the intervention and follow-up period.

\section{Conclusions}

Despite other accomplishments, the statewide implementation of Project Lazarus in North Carolina did not meet its objectives of marked increases in the utilization of buprenorphine or reductions in opioid analgesic prescribing. Future support for community coalitions addressing the opioid crisis may need a more narrow focus and targeted coalition capacity building to ensure impacts on such outcomes as prescribing behaviors and addiction treatment.

\section{Abbreviations \\ CCNC: Community Care of North Carolina; CSRS: North Carolina controlled substance reporting system; ED: Emergency Department; GEE: Generalized estimating equations; IRR: Incidence rate ratios; MAHEC: Mountain Area Health Education Center; MAT: Medication assisted treatment; MOR: Mu- opioid receptor; NC DHHS: North Carolina Department of Health and Human Services; NC: North Carolina; NC-TOPPS: North Carolina treatment outcomes and program performance system; OA: Opioid analgesics; ONDCP: Office of National Drug Control Policy; PDMP: Prescription Drug Monitoring Program; PL: Project Lazarus; SAMHSA: Substance Abuse and Mental Health Services Administration; SBI: North Carolina State Bureau of Investigation}

\section{Acknowledgements}

The authors thank a wide range of collaborators for funding, data, and expertise. The authors are grateful to coalitions and their leaders, CCNC Coordinators, local health departments, public health advocates, and other stakeholders who implemented the intervention, and are too numerous to name, but whose contributions were essential.

\section{Funding}

This evaluation study was funded by the United States Centers for Disease Control and Prevention (CDC; Cooperative Agreement 5U01CE002162-02), the Kate B. Reynolds Charitable Trust (KBR), a private foundation, and the Office of Rural Health (ORH), NC Department of Health and Human Services. KBR and ORH selected the order in which counties received funding for intervention implementation, but had no role in collection, management, analysis, and interpretation of the data; nor preparation, review, or approval of the manuscript; nor decision to submit the manuscript for publication.

\section{Availability of data and materials}

The data set used in analysis containing exposure and contextual variables is available for collaborative sharing upon request to the authors. For many variables, public data were used and the authors can direct interested parties to the original sources. Data on controlled substance prescriptions and drug treatment admissions can be made available for public use but require separate data use agreements directly with the NC Department of Health and Human Services, and cannot be disclosed by the authors without their written permission. Geographic identifiers for low population areas may be anonymized due to privacy concerns.

\section{Authors' contributions}

All authors were involved in study design. Data collection instruments were designed by AM, CLR, and ND. AM and AAA were directly involved in data collection. Analyses were conducted by AAA, ND, and SWM. AAA and ND had full access to all of the data used and take full responsibility for data integrity and the accuracy of the analysis. SWM, WR, PRC, CLR, and ND contributed to the development and revision of the manuscript. All authors have given final approval of the version to be published.

Ethics approval and consent to participate

Ethics approval was obtained through the Office of Human Reseach Ethics at the University of North Carolina at Chapel Hill (IRB 12-2570, 17-0889).

\section{Consent for publication}

Not applicable.

Competing interests

The authors declare that they have no competing interests.

\section{Publisher's Note}

Springer Nature remains neutral with regard to jurisdictional claims in published maps and institutional affiliations.

\section{Author details}

${ }^{1}$ Injury Prevention Research Center, University of North Carolina at Chapel Hill, Chapel Hill, North Carolina, USA. 'Department of Epidemiology, Gillings School of Global Public Health, University of North Carolina at Chapel Hill, Chapel Hill, North Carolina, USA. ${ }^{3}$ Department of Medicine, School of Medicine, University of North Carolina at Chapel Hill, Chapel Hill, North Carolina, USA.

Received: 25 June 2018 Accepted: 28 December 2018

Published online: 21 January 2019

\section{References}

Albert S, Brason FW 2nd, Sanford CK, Dasgupta N, Graham J, Lovette B. Project Lazarus: community-based overdose prevention in rural North Carolina. Pain Med. 2011;12(Suppl 2):S77-85.

Alexandridis AA, Dasgupta N, Ringwalt C, Sanford C, McCort A. Effect of local health department leadership on community overdose prevention coalitions. Drug \& Alcohol Depend. 2017;171:e5-6.

Alexandridis AA, McCort A, Ringwalt CL, Sachdeva N, Sanford C, Marshall SW, et al. A statewide evaluation of seven strategies to reduce opioid overdose in North Carolina. Inj Prev. 2018;24:48-54.

Blondell RD, Ashrafioun L, Dambra CM, Foschio EM, Zielinski AL, Salcedo DM. A clinical trial comparing tapering doses of buprenorphine with steady doses for chronic pain and co-existent opioid addiction. J Addict Med. 2010;4(3): 140-6.

Brinkley-Rubinstein L, Cloud DH, Davis C, Zaller N, Delany-Brumsey A, Pope L, et al. Addressing excess risk of overdose among recently incarcerated people in the USA: harm reduction interventions in correctional settings. Int J Prison Health. 2017;13(1):25-31.

Carrieri MP, Amass L, Lucas GM, Vlahov D, Wodak A, Woody GE. Buprenorphine use: the international experience. Clin Infect Dis. 2006;43(Supplement_4): S197-215.

Cepeda MS, Coplan PM, Kopper NW, Maziere J-Y, Wedin GP, Wallace LE. ER/LA opioid analgesics REMS: overview of ongoing assessments of its progress and its impact on health outcomes. Pain Med. 2017;18(1):78-85.

Christie C, Baker C, Cooper R, Kennedy PJ, Madras B, Bondi P. In: Office of National Drug Control Policy, editor. The President's commission on combating drug addiction and the opioid crisis. Washington, DC: The White House; 2017.

Ciccarone D. Fentanyl in the US heroin supply: a rapidly changing risk environment. Int J Drug Policy. 2017;46:107-11. 
Cicero TJ, Ellis MS, Harney J. Shifting patterns of prescription opioid and heroin abuse in the United States. N Engl J Med. 2015;373(18):1789-90.

Dart RC, Severtson SG, Bucher-Bartelson B. Trends in opioid analgesic abuse and mortality in the United States. N Engl J Med. 2015a;372(16):1573-4.

Dart RC, Surratt HL, Cicero TJ, Parrino MW, Severtson SG, Bucher-Bartelson B, et al. Trends in opioid analgesic abuse and mortality in the United States. N Engl J Med. 2015b;372(3):241-8.

Dasgupta N, Beletsky L, Ciccarone D. Opioid Crisis: No Easy Fix to Its Social and Economic Determinants. American Journal of Public Health 2018;108(2):182-86.

Delcher C, Wagenaar AC, Goldberger BA, Cook RL, Maldonado-Molina MM. Abrupt decline in oxycodone-caused mortality after implementation of Florida's prescription drug monitoring program. Drug Alcohol Depend. 2015;150:63-8.

Emmanuelli J, Desenclos JC. Harm reduction interventions, behaviours and associated health outcomes in France, 1996-2003. Addiction. 2005;100(11):1690-700.

Fiellin DA, Kleber H, Trumble-Hejduk JG, McLellan AT, Kosten TR. Consensus statement on office-based treatment of opioid dependence using buprenorphine. J Subst Abus Treat. 2004;27(2):153-9.

Fiellin DA, Schottenfeld RS, Cutter CJ, Moore BA, Barry DT, O'Connor PG. Primary care-based buprenorphine taper vs maintenance therapy for prescription opioid dependence: a randomized clinical trial. JAMA Intern Med. 2014; 174(12):1947-54.

Food and Drug Administration. Shared risk evaluation mitigation strategy for all immediate-release Transmucosal fentanyl dosage forms. J Pain Palliat Care Pharmacother. 2012;26(2):123-6.

Friedmann PD, Hoskinson R, Gordon M, Schwartz R, Kinlock T, Knight K, et al. Medication-assisted treatment in criminal justice agencies affiliated with the criminal justice-drug abuse treatment studies (CJ-DATS): availability, barriers, and intentions. Subst Abus. 2012;33(1):9-18.

Galea S, Vlahov D. Social determinants and the health of drug users: socioeconomic status, homelessness, and incarceration. Public Health Rep. 2002;117(Suppl 1):S135-S45.

Garcia MC, Dodek AB, Kowalski T, Fallon J, Lee SH, lademarco MF, et al. Declines in opioid prescribing after a private insurer policy change - Massachusetts, 2011-2015. MMWR Morb Mortal Wkly Rep. 2016;65(41):1125-31.

Injury and Epidemiology Surveillance Unit, Injury and Violence Prevention Branch, Division of Pubilc Health, North Carolina Department of Health and Human Services. Opiate Poisonings by Intent and County, 1999-2015. 2015.

Kegler MC, Rigler J, Honeycutt S. How does community context influence coalitions in the formation stage? A multiple case study based on the community coalition action theory. BMC Public Health. 2010;10(1):90

Kegler MC, Steckler A, Mcleroy K, Malek SH. Factors that contribute to effective community health promotion coalitions: a study of 10 project ASSIST coalitions in North Carolina. Health Educ Behav. 1998;25(3):338-53.

Knudsen HK, Abraham AJ, Oser CB. Barriers to the implementation of medicationassisted treatment for substance use disorders: the importance of funding policies and medical infrastructure. Eval Program Plann. 2011;34(4):375-81.

Kraus ML, Alford DP, Kotz MM, Levounis P, Mandell TW, Meyer M, et al. Statement of the American society of addiction medicine consensus panel on the use of buprenorphine in office-based treatment of opioid addiction. J Addict Med. 2011;5(4):254-63.

Lewis ET, Cucciare MA, Trafton JA. What do patients do with unused opioid medications? Clin J Pain. 2014;30(8):654-62.

Lipari RN, Hughes A. How people obtain the prescription pain relievers they misuse. CBHSQ Report 2017(Jan 12):1-7.

Mattick RP, Breen C, Kimber J, Davoli M. Buprenorphine maintenance versus placebo or methadone maintenance for opioid dependence. Cochrane Database Syst Rev. 2014;(2). Art. No.: CD002207.

North Carolina Department of Health and Human Services. North Carolina's opioid action plan, 2017-2021. 2017.

Remington PL, Catlin BB, Gennuso KP. The county health rankings: rationale and methods. Popul Health Metrics. 2015;13:11

Ringwalt C, Sanford C, Dasgupta N, Alexandridis A, McCort A, Proescholdbell S, et al. Community readiness to prevent opioid overdose. Health Promot Pract. 2018;19(5):747-55

State Center for Health Statistics. North Carolina vital statistics - deaths 20072014. V1 ed. Chapel Hill, NC: Odum Institute for Research in Social Science; 2015.

Thomas CP, Fullerton CA, Kim M, Montejano L, Lyman DR, Dougherty RH, et al. Medication-assisted treatment with buprenorphine: assessing the evidence. Psychiatr Serv. 2014;65(2):158-70.
Trado CE. Addressing pain management and palliative care: the official position of the North Carolina medical board. NC Med J. 2004;65(4):236-41.

Unick GJ, Rosenblum D, Mars S, Ciccarone D. Intertwined epidemics: national demographic trends in hospitalizations for heroin- and opioid-related overdoses, 1993-2009. PLoS One. 2013;8(2):e54496.

United States. National drug control strategy. Washington, D.C.: Office of National Drug Control Policy, Executive Office of the President; 2015.

Wakeland W, Nielsen A, Geissert P. Dynamic model of nonmedical opioid use trajectories and potential policy interventions. Am J Drug Alcohol Abuse. 2015;41(6):508-18.

Web-based Injury Statistics Query and Reporting System (WISQARS) [online] [Internet]. 2005.
Ready to submit your research? Choose BMC and benefit from:

- fast, convenient online submission

- thorough peer review by experienced researchers in your field

- rapid publication on acceptance

- support for research data, including large and complex data types

- gold Open Access which fosters wider collaboration and increased citations

- maximum visibility for your research: over $100 \mathrm{M}$ website views per year

At BMC, research is always in progress.

Learn more biomedcentral.com/submissions 